\section{Métodos de avaliação do consumo alimentar de gestantes: uma revisão}

\section{Review of methods of dietary assessment during pregnant}

Renata Labronici Bertin 1

Jane Parisenti 2

Patrícia Faria Di Pietro 3

Francisco de Assis Guedes de Vasconcelos 4
1-4 Programa de Pós-Graduação em Nutrição. Centro de Ciências da Saúde. Universidade de Santa Catarina. Campus Universitário. Trindade. Florianópolis, SC, Brasil. CEP: 99.040-970

E-mail: fguedes@floripa.com.br

1 Endereço: Rua Antônio Lago, 420, casa 9 Bairro Boa Vista/ Curitiba - Paraná - CEP: 82.560-470.

2,3,4 Endereço: Universidade Federal de Santa Catarina. Campus Universitário Trindade. Centro de Ciências da Saúde /Programa de Pós-Graduação em Nutrição.Florianópolis, SC, Brasil. CEP: 99.040-970.

\begin{abstract}
Physiological pregnancy changes impact nutritional needs and food intake. The adequate use of tools providing knowledge of food consumption during this life cycle is relevant because it enables the diagnosis for possible nutrition deficits and excesses. The objective of the survey was to perform a bibliographic review on food intake assessment methods during pregnancy. The literature reviewed was selected from an electronic database published between 1994 and September 2004 in Brazil and abroad. This article aims at describing and assessing the different methods and main results of studies determining food intake during pregnancy, among them, the following are highlighted: 24 hour recall, food registration, questionnaire on food intake consumption and food history. The results determine that the 24 hour recall method was the one more frequently used, nevertheless, for many times it was not applied beyond a two day investigation period and it did not take weekends into account. The choice for this method is related to pragmatism and a favorable cost benefit ratio. The conclusion is that to obtain reliable results, the choice of method and study design should always be related to the objectives of the enquiry.
\end{abstract}

Key words Food consumption, Pregnant women, Dietary enquiry.

\section{Resumo}

As alterações fisiológicas causadas pela gravidez. modificam as necessidades nutricionais $e$ a ingestão alimentar de gestantes. A utilização correta de instrumentos que proporcionem o conhecimento do consumo alimentar no decorrer deste ciclo de vida torna-se relevante por permitir diagnosticar possíveis carências e excessos nutricionais. $O$ objetivo da investigação foi realizar uma revisão bibliográfica sobre métodos de avaliação do consumo alimentar de gestantes. Os estudos analisados foram selecionados em bases eletrônicas de dados e publicados no período de 1994 a setembro de 2004, no Brasil e no exterior. Este artigo procura descrever e analisar os distintos métodos e os principais resultados dos estudos sobre determinação do consumo alimentar de gestantes, entre os quais destacam-se: recordatório de 24 horas $(R 24 h)$, registro alimentar, questionário de freqüencia de consumo alimentar e história alimentar. Os resultados apontam que o R24h foi o método mais freqüente, entretanto, seu uso muitas vezes não ultrapassa dois dias de investigação e nem leva em consideração finais de semana. A preferência por esse método está associada a sua praticidade e custo/benefício. Pode-se concluir que para obtenção de resultados seguros, a escolha do método e do desenho do estudo deve estar sempre relacionada com os objetivos da pesquisa.

Palavras-chave Consumo alimentar, Gestantes, Inquéritos dietéticos. 


\section{Introdução}

A gestação é uma fase muito importante na vida da mulher e requer alguns cuidados especiais. Os níveis de nutrientes nos tecidos e líquidos disponíveis para sua manutenção estão modificados por alterações fisiológicas (expansão do volume sangüíneo, alterações cardiovasculares, distúrbios gastrintestinais e variação da função renal) e por alterações químicas (modificações nas proteínas totais, lipídios plasmáticos, ferro sérico e componentes do metabolismo do cálcio). Por tais motivos faz-se necessária uma adequação na alimentação da gestante, visto que seu estado nutricional pode afetar o resultado da gravidez. 1-3 Para tanto, é de suma importância a avaliação dietética da gestante através de inquéritos alimentares, pois podemos detectar problemas nutricionais específicos já existentes, e que podem ser prejudiciais no decorrer da gestação. 4

Estudar o consumo alimentar humano é uma tarefa complexa, pois a alimentação envolve dimensões biológicas, socioeconômicas, culturais e simbólicas. Os dados coletados através de inquéritos dietéticos podem sofrer interferências de diferentes fatores relacionadas a essas distintas dimensões. Sendo assim, os inquéritos alimentares nem sempre fornecem informações precisas, em especial em indivíduos sujeitos a tratamentos dietéticos, os quais receberam informações sobre a alimentação adequada para seu estado de saúde. No caso específico das gestantes, sabe-se que as alterações do estado fisiológico e psicológico, muitas vezes podem influenciar os resultados de estudos de análise do consumo alimentar.5

Distintos métodos têm sido utilizados para determinação do consumo alimentar de gestantes, entre eles o recordatório de 24 horas (R24h), o registro alimentar (RA), o questionário de freqüência de consumo alimentar (QFCA) e a história alimentar (HA). Em linhas gerais, esses métodos podem ser assim sintetizados: o R24h consiste na obtenção, através de entrevista, de informações quantitativas dos alimentos e bebidas consumidos nas 24 horas precedentes ou no dia anterior, da primeira à última refeição do dia, caracterizando o consumo atual; o RA é um método onde o próprio indivíduo ou responsável anota as estimativas das porções de alimentos consumidos, seus tipos, receitas e preparações por um dia, uma semana ou um período mais longo, caracterizando o consumo atual; o QFCA é constituído por uma lista dos alimentos mais freqüentemente consumidos ou que formam o padrão alimentar da região, no qual se registra a freqüência habitual de consumo (nunca, diária, semanal, mensal etc.) e, finalmente, na história alimentar (HA) busca-se a obtenção de informações sobre o consumo e hábitos alimentares do indivíduo ao longo do seu ciclo de vida, podendo cobrir o período de um dia, uma semana, um mês ou período mais longo, possibilitando a caracterização do consumo habitual ou usual. ${ }^{6-9}$

As distintas modalidades de inquéritos dietéticos mostram-se importantes para avaliar o consumo alimentar habitual e atual de uma população. 10 No entanto, estudos utilizando o método da água duplamente marcada, considerado padrão-ouro para determinação do gasto energético, mostram importantes discrepâncias nos resultados dos inquéritos dietéticos. 10,11

Com a utilização do método da água duplamente marcada pode-se perceber que a investigação do consumo alimentar através de inquéritos dietéticos apresenta limitações e, de maneira geral, subestima a ingestão energética, em especial ao sub-relato do próprio indivíduo. 10,11 Segundo Bingham 12 parte deste erro deve-se também às tabelas de composição de alimentos que são limitadas para fornecer os reais valores de macro e micronutrientes consumidos. Comparando o método da água duplamente marcada com o R24h, por exemplo, observou-se um subrelato de aproximadamente $20 \%$ em relação à necessidade energética. Portanto, a inconsistência dos dados coletados através dos inquéritos dietéticos pode gerar vieses importantes em pesquisas, em especial na população obesa. Por outro lado, estudos bem conduzidos podem fornecer dados precisos do consumo alimentar, desde que os pesquisadores estejam bem treinados e os entrevistados motivados. 10

Este estudo tem por objetivo realizar uma revisão bibliográfica sobre métodos de avaliação do consumo alimentar de gestantes. Procurou-se identificar e analisar as distintas modalidades e os principais resultados de inquéritos sobre consumo alimentar de gestantes realizados no Brasil e no exterior.

\section{Métodos}

Realizou-se um levantamento bibliográfico em bases de dados eletrônicas com o objetivo de identificar estudos nacionais e internacionais, publicados no período de 1994 a setembro de 2004, que empregaram métodos de investigação dietética em gestantes. As bases eletrônicas consultadas foram: Scientific Eletronic Library On-line (SciELO), Literatura Latino-Americana e do Caribe em 
Ciências da Saúde (LILACS) e Medical Literature Analysis and Retrieval System Online (Medline) da National Library of Medicine. Os termos utilizados em português foram gestante, nutrição, mulher, consumo alimentar, inquérito alimentar, consumo dietético e seus correspondentes em inglês: pregnancy, nutrition, woman, food intake, food inquiry, dietary intake.

A Tabela 1 apresenta os artigos selecionados e analisados, segundo os autores e ano da publicação, as características da amostra, o método de investigação do consumo alimentar utilizado e os objetivos da aplicação do respectivo método. Foram selecionados quatorze artigos que empregaram métodos de investigação dietética em gestantes, sendo oito nacionais e seis internacionais. Nesses artigos, os métodos de investigação dietética utilizados foram: R24h, QFCA e RA. O método R24h foi o mais freqüentemente utilizado de forma isolada ou combinada com outro método, correspondendo a nove artigos ana-lisados. O QFCA foi o segundo mais freqüentemente utilizado, de forma isolada ou combinada, aparecendo em cinco artigos. O RA foi utilizado, isolado ou combinado, em dois artigos.

Os artigos selecionados foram analisados em relação ao tipo de inquérito utilizado, às características gerais da amostra, ao país de origem e aos objetivos da aplicação do método.

\section{Revisão dos estudos dietéticos realizados com gestantes}

Azevedo e Sampaio13 realizaram estudo com 99 adolescentes gestantes, entre 14 e 19 anos, em primeira visita de assistência pré-natal em um serviço público da cidade de Fortaleza, no Ceará. Foi aplicado um questionário socioeconômico e inquérito alimentar R24h, realizado em três dias não consecutivos, incluindo-se um dia de final de semana. O primeiro recordatório foi realizado no Serviço de Tocoginecologia Infanto-Puberal, antes da consulta de pré-natal, e os outros dois na residência da gestante. A análise foi efetuada utilizando-se a média dos três recordatórios. O critério de adequação do consumo alimentar adotado foi de $2500 \mathrm{kcal} /$ dia para energia e $60 \mathrm{~g} / \mathrm{dia}$ de proteínas. Pela análise dos recordatórios, observouse que $63,6 \%$ das gestantes apresentaram consumo inferior a $90 \%$ das necessidades energéticas, apenas $22,2 \%$ consumo entre $90 \%$ e $110 \%$ e as demais consumiam $110 \%$ das necessidades. A média de ingestão energética das adolescentes foi de 2347 kcal. A maioria das dietas revelou-se com conteúdo protéico adequado, excessivo em lipídeos e inadequado em carboidratos. A análise da adequação de micronutrientes, indicou que as dietas estavam muito inadequadas em relação ao cálcio, ferro, zinco, vitamina $\mathrm{B} 1$ e ácido fólico. Somente a vitamina $\mathrm{C}$ e a vitamina A apresentaram resultados satisfatórios. A deficiência da maioria dos micronutrientes pode ser explicada pelo baixo consumo de frutas, hortaliças, leite e seus derivados. Embora o consumo de frutas tenha sido baixo, as mais consumidas foram manga e acerola, o que pode explicar as vitaminas $\mathrm{C}$ e A acima das recomendações. Verificou-se pouca variação no cardápio diário, ocorrendo situações nas quais todos os três recordatórios foram praticamente iguais tanto no tipo quanto na quantidade dos alimentos consumidos.

O estudo realizado por Mikode e White, 14 no Reino Unido, com 20 mulheres grávidas, na faixa etária de 23 a 37 anos, pertencentes à classe média, avaliou a ingestão de nutrientes durante cada trimestre de gestação através de inquérito recordatório de 24 horas e registro alimentar. Foram realizados inquéritos dietéticos em cada trimestre da gestação, incluindo três $\mathrm{R} 24 \mathrm{~h}$ (o primeiro realizado no consultório médico no momento do diagnóstico da gravidez e dois por telefone) e dois RA de dois dias, os quais foram enviados por correio. Os instrumentos foram aplicados em dois períodos diferentes de cada trimestre incluindo um ou dois dias de final de semana. Foi observado aumento na ingestão de proteína, cálcio, zinco, riboflavina, folato, vitamina B6 e A, do primeiro para o segundo e do primeiro para o terceiro trimestre. Somente o zinco aumentou significantemente do segundo para o terceiro trimestre. A vitamina $\mathrm{C}$ diminuiu do segundo para o terceiro trimestre. A ingestão de proteína mostrou-se elevada em todos os trimestres. A ingestão de cálcio foi satisfatória em todos os trimestres. Somente o ferro apresentou ingestão abaixo de $66 \%$ da recomendação. A vitamina B6 e o folato também apresentaram baixa ingestão. Todos os bebês nasceram a termo, com peso normal. Embora as calorias inferidas fossem abaixo da recomendação, as mesmas se mostraram suficientes para manter as necessidades de energia para o ganho de peso entre a primeira e a última consulta pré-natal.

O estudo realizado por Dunn et al. 15 com 30 grávidas adolescentes (média de 16 anos) do Estado da Carolina do Norte, nos Estados Unidos, utilizaram um guia de entrevista com 48 itens elaborados após revisão da literatura. O guia tinha questões sobre características sócio-demográficas, hábitos dietéticos e alimentos preferidos e os que não gostam, sobre planejamento das refeições, 
Relação dos estudos sobre métodos de investigação do consumo alimentar de gestantes, publicados no período de 1994 a 2004, conforme autoria e ano de publicação, características gerais da população e amostra, método de investigação e objetivo da aplicação do método.

\begin{tabular}{ll} 
Autor/ano & Características da população e amostra \\
\hline Johnson et al.27 Washington (EUA), 1994. & $\begin{array}{l}\text { Gestantes americanas (afro-americanas), } \\
\text { nulíparas }-\mathrm{n}=744\end{array}$
\end{tabular}

Método de investigação do consumo alimentar

Recordatório 24 horas (aplicado mensalmente, durante o período da gravidez)

Mikode \& White14 (Reino Unido), 1994

Gestantes (23 a 37 anos) - $\mathrm{n}=20$

Dunn et al.15 Carolina do Norte (EUA), 1994 Gestantes adolescentes $-\mathrm{n}=30$

Schaffer et al.30 Califórnia (EUA), 1998

Gestantes americanas de origem latina e não latina - $\mathrm{n}=462$

Giddens et al.23 Ohio (EUA), 2000

Fujimori et al.22 São Paulo (Brasil), 2000

Camargo \& Veiga29 (Brasil) , 2000

Durán et al.16 Concepción, 2002

Nascimento \& Souza28 São Paulo (Brasil), 2002

Maeda31 São Paulo (Brasil), 2002

Villar \& Roncada25 São Paulo (Brasil), 2002

Azevedo \& Sampaio13 Fortaleza, (Brasil) 2003

Franceschini et al.20 São Paulo (Brasil),

2003

Barros et al.21 Rio de Janeiro (Brasil), 2004
Adolescentes no primeiro e segundo trimestre de gravidez $-\mathrm{n}=156$

(idade $<20$ anos) $\mathrm{n}=75$

Gestantes adolescentes -

(12 a 18,9 anos) $n=140$

Gestantes $\geq 20$ anos $n=146$

Gestantes com sobrepeso $-\mathrm{n}=110$

Gestantes (março de 1997 março de 1998) $n=14$

Gestantes (idade entre $19 \mathrm{e}$ 40 anos) - $n=91$

Gestantes adolescentes $-\mathrm{n}=99$

Gestantes $-\mathrm{n}=77$

Gestantes adolescentes $-\mathrm{n}=1.180$

\section{Gestantes adolescentes -}

Recordatório de 24 horas ( 2 dias em cada trimestre, incluindo 1 ou 2 de final de semana) e registro alimentar.

Guia de entrevista (hábitos dietéticos,

alimentos preferidos, aversões, planejamento estado nutricional. das refeições, compra e preparo de alimentos) e recordatório de 24 horas

Questionário de freqüência alimentar com 100 itens

2 registros alimentares de 7 dias (14 dias)

Questionário de consumo de Freqüência Alimentar

Recordatório de 24 horas e QFCA

Recordatório de 24 horas (2 dias consecutivos)

Recordatório de 24 horas (1 dia)

Questionário Quantitativo de Freqüência Alimentar (QQFA) em 3 momentos e Índice de Qualidade da Dieta Saudável adaptado à gestantes (IQDSg)

Formulário Dietético Simplificado (FDS) (recordatório 24 horas e questionário de freqüência de consumo)

Recordatório de 24 horas (3 dias não consecutivos)

Recordatório de 24 horas
Objetivo da aplicação do método

Relacionar o consumo alimentar e

composição corporal no pré-natal e pósparto.

Avaliar a ingestão de nutrientes em cada trimestre da gestação.

Avaliar o consumo alimentar e práticas saudáveis.

Examinar o consumo dietético de adolescentes grávidas e comparar com gestantes adultas.

Avaliação da ingestão de ferro.

Avaliar a ingestäo alimentar de adolescentes gestantes.

Quantificar o consumo de sódio, potássio e calcio e relacionar com a pressão arterial.

Avaliar o consumo alimentar e as possíveis deficiências nutricionais.

Avaliar o padrão de consumo de alimentos o ganho ponderal durante a gestação em mulheres de baixa renda.

Avaliação do consumo de vitamina A em gestantes.

Analisar o consumo alimentar

Verificar a influência do estado nutriciona materno com o peso ao nascer

Verificar possíveis deficiências nutricionais 
compra e preparação dos alimentos e as pacientes também responderam um R24h. A maioria das adolescentes ultrapassou a recomendação energética, de proteínas e gorduras (em especial as saturadas), mas a maioria não atingiu a necessidade de ferro e fibras. Foi observada baixa ingestão de frutas e vegetais.

Duran et al., 16 em investigação com o objetivo de quantificar o consumo de sódio, potássio e cálcio, em gestantes normotensas da área urbana de Concepción, no Chile, entrevistaram 146 gestantes com 20 anos ou mais e sem patologias associadas. As informações nutricionais foram obtidas através de R24h, em dois dias consecutivos. Para obter a disponibilidade de sal diária por pessoa, registrou-se a compra mensal estimada de sal. A pressão arterial foi medida por duas vezes, com intervalo de 10 minutos, em duas ocasiões. Os resultados mostraram variação entre um inquérito e outro quanto ao sódio e especialmente potássio. Quanto às calorias, se notou pouca variação. O R24h de dois dias apresenta probabilidade de erro, de $30 \%$ nos valores de aportes calóricos; de $40 \%$ nos valores da ingestão de sódio e uma chance de erro de $50 \%$ para o potássio e o cálcio. Dessa forma, os autores recomendam aumentar os dias de registro dietético e, além disso, considerar os alimentos consumidos fora de casa (restaurantes, lanchonetes, snacks, bares, o sal adicionado à mesa, temperos, e o sal adicionado no processamento industrial dos alimentos), para a determinação mais precisa do padrão alimentar da população. Vale ressaltar que estudo realizado por Dyer et al.,17 mostrou que cerca de $75 \%$ do sal consumido em países industrializados provém de alimentos processados. Para diminuir o erro na estimação da ingestão, alguns autores ${ }^{18,19}$, sugerem um estudo com RA com mais de sete dias consecutivos complementado com a excreção urinária dos minerais envolvidos.

Franceschini et al.,20 ao avaliarem os fatores de risco para o baixo peso ao nascer em 77 gestantes (idade entre 15 a 48 anos), residentes em favelas do município de São Paulo, utilizaram o R24h para verificar a influência do estado nutricional materno com o peso ao nascer do concepto, o qual foi aplicado uma única vez. Através do recordatório, foi identificado que o consumo energético era insuficiente para que o ganho de peso gestacional e o ganho de peso do bebê atingissem a faixa de normalidade. A partir do estado nutricional e do recordatório foi realizada orientação nutricional.

Barros et al.21 investigaram o consumo alimentar de 1180 puérperas adolescentes, com idades entre 12 e 19 anos, no município do Rio de Janeiro.
Descreveram os alimentos mais consumidos, a ingestão diária de energia, proteína, ferro, vitamina $\mathrm{C}$, folato e cálcio, através de dois questionários aplicados no pós-parto imediato. O primeiro questionário foi aplicado a todas as puérperas e constava de perguntas sobre as condições socioeconômicas, história reprodutiva e utilização de serviços de saúde. O segundo questionário, específico para as adolescentes, abordava as relações familiares, estilo de vida e a alimentação durante a gravidez. Para avaliação do consumo alimentar, foi utilizado um QFCA composto por uma lista com 23 alimentos, semiquantitativo, em porções padronizadas. A lista de alimentos do QFCA foi obtida com base no questionário utilizado na investigação do consumo de alimentos da população do Rio de Janeiro, desenvolvida na pesquisa de base populacional "Nutrição e Saúde no Rio de Janeiro", realizada em 1995-1996. Para estimar o consumo diário de energia, proteína, ferro, vitamina $\mathrm{C}$, folato e cálcio, transformaram cada freqüência relatada em frequiência diária. Os alimentos mais freqüentemente consumidos foram arroz, açúcar, manteiga, pão, feijão, leite, refrigerante, sucos, legumes e laranja, sendo que nas adolescentes menores de 15 anos o consumo de sucos, legumes e frutas diminuíram. Tal estudo possibilitou identificar os alimentos mais consumidos na dieta das adolescentes durante a gravidez $\mathrm{e}$ as possíveis deficiências nutricionais. Verificou-se um baixo consumo de energia para cerca de metade das adolescentes, baixo aporte de ferro, folato e cálcio. $\mathrm{O}$ consumo da proteína foi adequado quanto ao consumo de vitamina $\mathrm{C}$, coerente com a frequiência de ingestão de sucos de frutas, laranjas e legumes (os quais estiveram entre os dez alimentos mais referidos pelas mães adolescentes), excedeu a ingestão recomendada.

Fujimori et al. 22 pesquisaram uma amostra de 75 gestantes adolescentes, com idade inferior a 20 anos, no município de Santo André, São Paulo, com o objetivo de caracterizar o estado nutricional de ferro, de acordo com as condições socioeconômicas, antecedentes familiares e pessoais, dados antropométricos, e a ingestão de ferro. Para avaliar a ingestão de ferro, foi utilizado um QFCA, onde os alimentos estavam distribuídos em grupos, os quais deveriam ser checados se consumidos ou não no dia anterior à consulta. Através do levantamento do consumo alimentar verificou-se que $96 \%$ ingeriram alimentos do grupo das carnes, ovos e leguminosas, diariamente, seguido pelo grupo de cereais, referido por $92 \%$ das gestantes; Cinquenta e seis porcento das gestantes ingeriram alimentos do grupo de leite e derivados e do grupo de hortaliças. O grupo das frutas 
foi consumido por $52 \%$ das gestantes e $38,7 \%$ ingeriram alimentos do grupo açúcares, doces e gorduras. Aqueles autores concluíram que a forma de analisar o consumo alimentar das gestantes não permitiu uma análise mais detalhada sobre a quantidade de ferro ingerido pelas mesmas. Entretanto, os dados levantados indicam que cerca de dois terços das gestantes $(64 \%)$ que consumiam o grupo das carnes, ovos e leguminosas, ingeriam carne bovina e feijão, sendo este último, importante fonte de ferroheme e estimulante da absorção de ferro não-heme presente nos alimentos de origem vegetal.

Giddens et al. 23 realizaram um estudo comparativo entre 59 gestantes adolescentes e 97 gestantes adultas do Estado de Ohio, Estados Unidos, cujo objetivo foi analisar o consumo energético e de nutrientes da dieta de gestantes adolescentes (idades de 13 a 18 anos) e comparar com a dieta de gestantes adultas (idades de 19 a 40 anos), enfocando as diferenças encontradas. Para a realização do estudo, foram utilizados 2 RA de sete dias, sendo o primeiro aplicado no segundo trimestre de gestação e o segundo no terceiro. Os autores não encontraram diferença nos valores de nutrientes entre o primeiro e o segundo registros e observaram que o consumo energético reportado pelas adolescentes foi superior ao encontrado pelas gestantes adultas, porém a distribuição de energia, entre os nutrientes (macro e micronutrientes) foi similar nos dois grupos. A média do consumo de cálcio, magnésio, zinco, ferro, fibra, folato, vitaminas D e E, em ambos os grupos foi inferior ao recomendado, enquanto que a média do consumo de proteína, tiamina, riboflavina, niacina, fósforo, selênio, vitamina B6, C e A foi atingida ou até ultrapassou os valores recomendados em ambos os grupos. Os autores concluem que o baixo consumo de nutrientes existe tanto em dieta de gestantes adolescentes quanto em adultas, indicando a necessidade de um suporte nutricional mais adequado, observando não só o consumo alimentar, mas também respeitando o estilo de vida de ambos os grupos.

O International Vitamin A Consultative Group (IVACG) desenvolveu o Formulário Dietético Simplificado (FDS) para determinar o risco de deficiência de Vitamina A. O FDS incluiu um R24h, para caracterizar o Índice de Consumo (IC) e um QFCA, cujo objetivo foi estabelecer o Padrão Usual de Consumo (PUC). ${ }^{24}$

Com o propósito de adaptar o FDS para gestantes e avaliar a concordância entre os instrumentos que compõem o FDS, Villar e Roncada25 desenvolveram um estudo com 91 gestantes (19 - 40 anos), da cidade de São Paulo. O instrumento FDS foi aplicado uma única vez, sendo utilizado um álbum com 126 fotografias para auxiliar na determinação do tamanho das porções pelas gestantes. Os autores concluíram que as gestantes estudadas apresentaram alto e moderado risco de deficiência de Vitamina A. Também concluíram que o instrumento foi válido na determinação do risco de deficiência do referido nutriente, mas sugeriram maior número de aplicações do IC.

Scagliusi e Lancha Júnior, 11 em um estudo de revisão da literatura, apontaram como possíveis fontes de erros dos inquéritos dietéticos o estado nutricional do indivíduo (obesos tendem a subrelatar o consumo alimentar mais que eutróficos), sexo (mais chances de sub-relato entre as mulheres), idade (quanto maior a idade, maior o erro), atividade física (sedentários apresentam menor sub-relato), região de moradia, nível educacional, classe social, fatores morais e emocionais, grau de informação e preocupação com a saúde, descontrole alimentar, fome e apetite, ajuste social (falar o que é socialmente correto) e atitude alimentar (busca pela magreza, dietas saudáveis). Para os autores, o subrelato parece ser mais significante para alimentos que são fontes de lipídeos e carboidratos, enquanto que a super-estimação é mais freqüente para frutas e verduras.

Além disso, Pereira e Koifman 26 observaram que a fidedignidade dos dados coletados através de inquéritos dietéticos não está simplesmente relacionado à escolha correta do método, e que também deve-se considerar que o relato é um processo mental que envolve valores e símbolos.

\section{Considerações finais}

Considerando que a gestação é um período onde ocorrem importantes modificações fisiológicas e psicológicas, bem como mudanças das necessidades nutricionais da mulher, deve-se buscar avaliar o consumo alimentar durante as fases do período gestacional, para assim obter dados mais fidedignos.

Ao analisar os inquéritos dietéticos empregados na população de gestantes (Tabela 1), observa-se que dos 14 estudos analizados, cinco realizaram a avaliação do consumo alimentar apenas pelo método do R24h,13,16,20,27,28 dois associaram o R24h e QFCA, 25,29 um utilizou o R24h combinado com o RA, 14 três utilizaram o QFCA, ${ }^{21,22,30}$ um apenas o $\mathrm{RA}, 23$ um estudo utilizou um guia sobre consumo e hábitos alimentares incluindo o R24h15 e um utilizou um Questionário Quantitativo de Freqüência Alimentar (QQFA) em três momentos e o Índice de 
Qualidade da Dieta Saudável adaptado a gestantes (IQDSg). 31

Diante dos vários métodos de avaliação do consumo alimentar, o R24h foi o mais utilizado. A preferência por esse método está associada a sua praticidade (curto tempo de aplicação) e custo/benefício. No entanto, o que observamos nos estudos foi que a maioria dos $\mathrm{R} 24 \mathrm{~h}$, muitas vezes não ultrapassam dois dias e não levam em consideração finais de semana e estações do ano, e mesmo assim contemplam o consumo de micronutrientes específicos. Segundo Bingham, 12 recomenda-se o emprego do método R24h por três dias para avaliar o total da ingestão de alimentos e nutrientes, sendo esse método capaz de estimar até mesmo as diferenças entre grupos de indivíduos em pesquisas epidemiológicas.

\section{Referências}

1. Picciano MF. Embarazo y lactancia. In: Ziegler EE, Filer JL, editores. Conocimientos actuales sobre nutrición. 7. ed. Washington (DC): OPAS, OMS; 1997. p. 410-22.

2. Cathy F. Nutrição durante a gravidez e a lactação In: Mahan LK, Escot-Stump S, editores. Alimentos, nutrição e dietoterapia. 10. ed. São Paulo: Roca; 2002. p. 159-86.

3. Paoli IR, Sánchez AA, Pérez GH. Cambios en las variables hematológicas y bioquímicas durante la gestación en mujeres eutróficas. An Venez Nutr 2002; 15:11-7.

4. Forsythe HE, Gage B. Use of multicultural food-frequency questionnaire with pregnant and lactating women. Am J Clin Nutr. 1994; 59 (Suppl): 203S-6S.

5. Garcia RWD. Representações sobre consumo alimentar e suas implicações em inquéritos alimentares: estudo qualitativo em sujeitos submetidos à prescrição dietética. Rev Nutr. 2004; 17: 15-28.

6. Gibson RS. Principles of nutritional assessment. New York: Oxford University Press; 1990.

7. Gouveia ELC. Nutrição, saúde \& comunidade. 2. ed. Rio de Janeiro: Revinter; 1999.

8. Vasconcelos FAG. Avaliação nutricional de coletividades. 3. ed. Florianópolis: Ed. da Universidade Federal de Santa Catarina; 2000.

9. Willett W. Nutritional epidemiology. 2. ed. New York: Oxford University Press; 1998.

10. Bellisle F. O método da água duplamente marcada e pesquisas de ingestão alimentar: um confronto. Rev Nutr 2001; 14: 125-33.

11. Scagliusi FB, Lancha Júnior AH. Subnotificação da ingestão energética na avaliação do consumo alimentar. Rev Nutr 2003; 16: 471-81.

12. Bingham SA. The dietary assessment of individuals: methods, accuracy, new thechniques and recommendations. Nutr Abstr Rev 1987; 57: 705-42
Para o Food and Nutrition Board, Nacional Research Council, ${ }^{32}$ para que se possa mensurar com mais fidedignidade o consumo usual dos nutrientes (macro e micronutrientes) por esse método, deve-se aplicar quatro recordatórios no mesmo indivíduo em um ano, considerando, finais de semana, estações do ano e férias. No entanto para muitas pesquisas fica inviável seguir esses critérios em função do tempo disponível para pesquisa.

Assim, para que haja uma melhor mensuração da ingestão alimentar através do R24h, é aconselhável o uso concomitante com outro inquérito dietético. 33

Para obtenção de resultados seguros, a escolha do método e do desenho do estudo deve estar sempre relacionada com os objetivos da pesquisa, mas muitas vezes as impossibilidades técnicas, financeiras e temporais determinam a escolha do método.

13. Azevedo DV, Sampaio HAC. Consumo alimentar de gestantes adolescentes atendidas em serviço de assistência pré-natal. Rev Nutr 2003; 16: 273-80.

14. Mikode MS, White A. Dietary assessment of middleincome pregnant women during the first, second, and third trimesters. J Am Diet Assoc 1994; 94: 196-9.

15. Dunn C, Kolasa K, Dunn PC, Ogle MB. Dietary intake of pregnant adolescents in a rural southern community. J Am Dietet Assoc 1994; 94:1040-1.

16. Durán EF, Soto DA, Asenjo AI, Labraña AM, Quiróz VG, Pradenas FP. Ingesta dietaria de sodio, potasio y calcio en embarazadas normotensas. Rev Chil Nutr 2002; 29: 40-5.

17. Dyer A, Elliott P, Chee D, Stamler J. Urinary biochemical markers of dietary intake in the INTERSALT Study. Am J Clin Nutr 1997; 65 (suppl): 1246S-53S.

18. Howat M, Mohan R, Champagne C, Monlezun C, Wozniak P, Bray G.Validity and reliability of reported dietary intake data. J Am Diet Assoc 1994; 94: 169-73.

19. Domas J, Tangney C, Rosenson S. Determination of the minimum number of food records required to assess nutrient intake accurately. Am J Clin Nutr 1997; 65 (suppl): 1327-30

20. Franceschini SCC, Priore SE, Pequeno NPF, Silva DG, Sigulen DM. Fatores de risco para o baixo peso ao nascer em gestantes de baixa renda. Rev Nutr 2003; 16: 171-9.

21. Barros DC, Pereira RS, Gama SGN, Leal MC. O consumo alimentar de gestantes adolescentes no Município do Rio de Janeiro. Cad Saúde Pública 2004; 20 (Supl 1): S121-S9.

22. Fujimori E, Laurent D, Cassana LMN, Oliveira IMV, Szarfarc SC. Anemia e deficiência de ferro em gestantes adolescentes. Rev Nutr PUCCAM 2000; 13: 177-84.

23. Giddens JB, Krug SK, Tsang RC, Guo S, Miodovnik M, Prada JA. Pregnant adolescent and adult women have similarly low intakes of selected nutrientes. J Am Diet Assoc 2000; 100: 1334-40. 
24. Sommer A, McLaren DS, \& Olson JA. Assessment of vitamin A status. IVACG Task Force on Assessment. In: Guidelines for the eradication of vitamin A deficiency and xerophthalmia; a report of the International Vitamin A Consultative Group (IVACG). New York, The Nutrition Foundation, 1976. p.I-1-I-7.

25. Villar SB, Roncada MJ. Determinação do consumo de alimentos fontes de vitamina A por gestantes, utilizando o formulário dietético simplificado (FDS). Archs Latinoam Nutr 2002; 52: 48-54.

26. Pereira RA, Koifman S. Uso do questionário de freqüência na avaliação do consumo alimentar pregresso. Rev Saúde Pública 1999; 33: 610-21.

27. Johnson AA, Knight, EM, Edwards CH, Oyemade UJ, Cole OJ, Westney OE, et al. Dietary intakes, anthropometric measurements and pregnancy outcomes. J Nutr 1994; 94 : 936S-942S.

28. Nascimento E, Souza SB. Avaliação da dieta de gestantes com sobrepeso. Rev Nutr 2002; 15: 174-9.

Recebido em 26 de julho de 2005

Versão final apresentada em 7 de novembro de 2006

Aprovado em 20 de novembro de 2006
29. Camargo RMS, Veiga GV. Ingestão e hábitos alimentares de adolescentes gestantes. Folha Med 2000; 119: 37-46.

30. Schaffer DM, Velie EM, Shaw GM, Todoroff KP. Energy and nutrient intakes and health practices of Latinas and non-Latinas in the three months before pregnancy. J Am Diet Assoc 1998; 98: 876-884.

31. Maeda AP. Padrão de consumo alimentar de gestantes atendidas em um serviço público de pré-natal: um estudo de coorte [dissertação mestrado]. São Paulo: Departamento de Nutrição da Faculdade de Saúde Pública da Universidade de São Paulo; 2002.

32. NRC (National Research Council). Recommended dietary allowances. 9.ed. Washington, D.C., National Academy Press, 1980.

33. Vitolo MR. Avaliação nutricional da gestante. In: Nutrição: da gestação à adolescência. Rio de Janeiro: Reichmann; 2003. p.18-29. 